\title{
Multiplicity and Semicontinuity of the Łojasiewicz Exponent
}

by

\section{Tomasz RODAK, Adam RÓŻYCKI and Stanisław SPODZIEJA}

\author{
Presented by Józef SICIAK
}

Summary. We give an effective formula for the improper isolated multiplicity of a polynomial mapping. Using this formula we construct, for a given deformation of a holomorphic mapping with an isolated zero at zero, a stratification of the space of parameters such that the Łojasiewicz exponent is constant on each stratum.

Introduction. Let $f:\left(\mathbb{C}^{n}, 0\right) \rightarrow\left(\mathbb{C}^{m}, 0\right)$ be a germ of a holomorphic map with an isolated zero. Then a lot of numerical invariants can be associated with this map. In this note we are interested in two of them: multiplicity and Eojasiewicz exponent.

The multiplicity of $f$ may be defined in several ways. Probably the best known is the notion of Hilbert-Samuel multiplicity (see [5]). Let $I$ be the ideal generated by the components of $f$ in the local ring $\left(\mathcal{O}_{n}, \mathfrak{m}_{n}\right)$ of germs of holomorphic functions $\left(\mathbb{C}^{n}, 0\right) \rightarrow \mathbb{C}$. Then the Hilbert-Samuel multiplicity of $I$ is the normalized leading coefficient of the Hilbert-Samuel polynomial of $I$; in our case it is given by the formula

$$
e(I)=\lim _{k \rightarrow \infty} \frac{n !}{k^{n}} \operatorname{dim} \mathcal{O}_{n} / I^{k} .
$$

If $f$ is a system of parameters (i.e. $m=n$ ), then

$$
e(I)=\operatorname{dim} \mathcal{O}_{n} / I \text {. }
$$

Moreover, in this case $e(I)$ has a well known geometric description: $e(I)=$ $i_{0}(f)$ where $i_{0}(f)$ is the number of points in the generic fiber of $f$. Using

2010 Mathematics Subject Classification: 32B10, 14Q99, 12 Y99.

Key words and phrases: multiplicity, effective formula, Łojasiewicz exponent.

Received 6 November 2015; revised 28 February 2016.

Published online 18 March 2016. 
results of R. Achilles, P. Tworzewski and T. Winiarski [1], it is possible to extend the geometric definition of $i_{0}(f)$ to the case $m>n$. Namely, let $i_{0}(f)$ be the improper intersection multiplicity of the graph of $f$ and $\mathbb{C}^{n} \times\{0\} \subset \mathbb{C}^{n} \times \mathbb{C}^{m}$ at the point $(0,0) \in \mathbb{C}^{n} \times \mathbb{C}^{m}$. In the case $m=n$ this notion was defined by R. Draper [2] (see also [12], [15]). In fact, with this generalization the multiplicity $i_{0}(f)$ is still equal to $e(I)$. Indeed, let $L: \mathbb{C}^{m} \rightarrow \mathbb{C}^{n}$ be a generic linear map. By [11] we have $i_{0}(f)=i_{0}(L \circ f)$ (see Theorem 1 below). On the other hand, the ideal generated by $L \circ f$ in $\mathcal{O}_{n}$ is a reduction of $I$, hence has the same Hilbert-Samuel multiplicity [5, Theorems 14.13, 14.14]. In what follows, we will denote the multiplicity of $f$ by $i_{0}(f)$.

Let us now proceed to the second invariant. Since $f$ is analytic, there exist $C>0$ and $\nu \geq 1$ such that

$$
|f(z)| \geq C|z|^{\nu}
$$

in some neighbourhood of the origin in $\mathbb{C}^{n}$. By definition, the Eojasiewicz exponent of $f$, denoted by $\mathcal{L}_{0}(f)$, is the infimum of the exponents $\nu$ in the above inequality. In [3] it was proved that $\mathcal{L}_{0}(f)$ is a rational number and the infimum is in fact a minimum. Moreover, in [3] an algebraic formula for the Łojasiewicz exponent was given:

$$
\mathcal{L}_{0}(f)=\inf \left\{\frac{p}{q}: \mathfrak{m}_{n}^{p} \subset \overline{I^{q}}\right\},
$$

where for any ideal $J$ in $\mathcal{O}_{n}, \bar{J}$ denotes the integral closure of $J$ in $\mathcal{O}_{n}$.

Now, let $h:\left(\mathbb{C}^{n}, 0\right) \rightarrow(\mathbb{C}, 0)$ be a germ of a holomorphic function defining an isolated singularity at $0 \in \mathbb{C}^{n}$ (i.e. the gradient $\nabla h$ of $h$ has an isolated zero). Then $\mu:=i_{0}(\nabla h)$ is the Milnor number of $h$. In [13], B. Teissier proved that if $s \mapsto h_{s}$ is an analytic family of functions with isolated singularities with constant Milnor number, then the function $s \mapsto \mathcal{L}_{0}\left(\nabla h_{s}\right)$ is lower semicontinuous. Moreover, he showed that if we do not assume that this family is $\mu$-constant then $\mathcal{L}_{0}(\nabla h)$ is neither upper nor lower semicontinuous [14]. The above result was generalized by A. Płoski [7 in the following way: If $s \mapsto f_{s}$ is an analytic family of holomorphic maps $\left(\mathbb{C}^{n}, 0\right) \rightarrow\left(\mathbb{C}^{n}, 0\right)$ with an isolated zero and of constant multiplicity, then the function $s \mapsto \mathcal{L}_{0}\left(f_{s}\right)$ is lower semicontinuous.

One may consider a further generalization of this result. Since the multiplicity $i_{0}$ is well defined for ideals which are not generated by a system of parameters, it is reasonable to ask if this assumption in the above result of Płoski is necessary. It was proved in [8] that it is enough to assume that the $f_{s}$ are maps $\left(\mathbb{C}^{n}, 0\right) \rightarrow\left(\mathbb{C}^{m}, 0\right)$ with $m$ possibly greater than $n$, with isolated zero of constant multiplicity. Under these assumptions the function $s \mapsto \mathcal{L}_{0}\left(f_{s}\right)$ is lower semicontinuous. 
In the paper we prove that for a given finite complex stratification $\left\{\Gamma_{\nu}^{i}\right\}$ of the space of parameters such that $f_{s}$ is multiplicity-constant on each stratum $\Gamma_{\nu}^{i}$, the function $s \mapsto \mathcal{L}_{0}\left(f_{s}\right)$ is lower semicontinous on this stratum and there exists a refinement $\left\{\Sigma_{\mu}^{j}\right\}$ of $\left\{\Gamma_{\nu}^{i}\right\}$ such that the function $s \mapsto \mathcal{L}_{0}\left(f_{s}\right)$ is constant on each stratum $\Sigma_{\mu}^{j}$ (Theorem 7). The proof is based on an algorithm which allows us to effectively compute the multiplicity $i_{0}(f)$ (Theorem 4. cf. [10]). As a corollary we get the above-mentioned semicontinuity theorem (Corollary 11).

1. A formula for multiplicity. Let $f:\left(\mathbb{C}^{n}, 0\right) \rightarrow\left(\mathbb{C}^{m}, 0\right)$ be a holomorphic mapping with an isolated zero. Denote by $\mathbb{L}(m, n)$ the set of all linear mappings $\mathbb{C}^{m} \rightarrow \mathbb{C}^{n}$.

The basis for our further considerations is

Theorem 1 ([11, Theorem 1.1]). For any $L \in \mathbb{L}(m, n)$ such that the mapping $L \circ f:\left(\mathbb{C}^{n}, 0\right) \rightarrow\left(\mathbb{C}^{n}, 0\right)$ has an isolated zero we have

$$
i_{0}(f) \leq i_{0}(L \circ f) .
$$

Moreover, for generic $L \in \mathbb{L}(m, n)$, the mapping $L \circ f$ has an isolated zero and

$$
i_{0}(f)=i_{0}(L \circ f) .
$$

The next proposition will be used to pass from holomorphic to polynomial germs of mappings.

Proposition 2 ([6, 11]). We have

$$
\mathcal{L}_{0}(f) \leq i_{0}(f) .
$$

Moreover, if $g:\left(\mathbb{C}^{n}, 0\right) \rightarrow\left(\mathbb{C}^{m}, 0\right)$ is a holomorphic mapping such that $\operatorname{ord}(f-g)>\mathcal{L}_{0}(f)$ then $g$ has an isolated zero and

$$
\mathcal{L}_{0}(g)=\mathcal{L}_{0}(f) \quad \text { and } \quad i_{0}(g)=i_{0}(f) .
$$

From now on we will assume that $f=\left(f_{1}, \ldots, f_{m}\right): \mathbb{C}^{n} \rightarrow \mathbb{C}^{m}$ is a polynomial mapping such that $0 \in \mathbb{C}^{n}$ is an isolated point of $f^{-1}(0)$.

Proposition 3 ([6, 11]). Let $d_{j}=\operatorname{deg} f_{j}, j=1, \ldots, m$. Assume that $d_{1} \geq \cdots \geq d_{m}$. Then

$$
\mathcal{L}_{0}(f) \leq d_{1} \cdots d_{n}
$$

The algorithm which computes $i_{0}(f)$ is given in the following construction.

Let $d=\max \left\{\operatorname{deg} f_{1}, \ldots, \operatorname{deg} f_{m}\right\}$. Define a mapping $H_{L}: \mathbb{C}^{n} \rightarrow \mathbb{C}^{n}$ by

$$
H_{L}(z)=L(f(z))+\left(z_{1}^{d^{n}+1}, \ldots, z_{n}^{d^{n}+1}\right),
$$

where $L \in \mathbb{L}(m, n)$. Set

$$
\mathbb{M}(m, n)=\mathbb{L}(m, n) \times \mathbb{L}(n, 1) \times \mathbb{C}^{n}
$$


and let

$$
\Phi: \mathbb{M}(m, n) \rightarrow \mathbb{M}(m, n) \times \mathbb{C}
$$

be given by

$$
\Phi(L, N, z)=\left(L, N, H_{L}(z), N(z)\right) .
$$

The mapping $\Phi$ is proper and consequently $\Phi(\mathbb{M}(m, n))$ is an algebraic set of pure dimension $m n+2 n$. So, there exists an irreducible polynomial $P \in \mathbb{C}[L, N, y, t]$, where $y=\left(y_{1}, \ldots, y_{n}\right)$ and $y_{1}, \ldots, y_{n}, t$ are independent variables, of the form

$$
P(L, N, y, t)=\sum_{j=0}^{p} P_{j}(L, N, y) t^{j}
$$

such that $P_{p} \neq 0$ and $\Phi(\mathbb{M}(m, n))=P^{-1}(0)$. Since $P$ vanishes exactly on the image of the polynomial map $\Phi$, it could be computed by means of Gröbner bases.

THEOREM 4. We have

$$
i_{0}(f)=\min \left\{j \in \mathbb{Z}: \operatorname{ord}_{y} P_{j}=0\right\} .
$$

The right hand side above is well defined in view of the following proposition, which is a special case of [9, Theorem 7].

Proposition 5. There exists $r \in \mathbb{Z}$ with $0 \leq r<p$ such that

$$
\operatorname{ord}_{y} P_{j}>0 \quad \text { for } j=0, \ldots, r \quad \text { and } \operatorname{ord}_{y} P_{r+1}=0 \text {. }
$$

Set

Then

$$
\Delta(P)=\min _{j=0}^{r} \frac{\operatorname{ord}_{y} P_{j}}{r+1-j}
$$

$$
\mathcal{L}_{0}(f)=\frac{1}{\Delta(P)}<d^{n}+1 .
$$

We will also use this proposition in the proof of the main result in the next section.

Proof of Theorem 4. Let $r$ be the integer given in Proposition5. We must prove that $i_{0}(f)=r+1$. Observe that there exists a Zariski open, nonempty set $\mathcal{U} \subset \mathbb{L}(m, n) \times \mathbb{L}(n, 1)$ such that if $(L, N) \in \mathcal{U}$ then:

- $L \circ f$ has an isolated zero at the origin,

- condition (5) is satisfied,

- $\left.N\right|_{H_{L}^{-1}(y)}$ is injective for generic $y \in \mathbb{C}^{n}$,

- $H_{L}^{-1}(0) \cap \operatorname{ker} N=\{0\}$.

Fix $(L, N) \in \mathcal{U}$. Then

$$
i_{0}\left(H_{L}\right)=r+1 .
$$

Indeed, by (5) the polynomial $P_{L, N}(y, t)$ is a $t$-regular function of order $r+1$. 
Using the Weierstrass preparation theorem we may write

$$
P_{L, N}(y, t)=Q_{L, N}(y, t) \widetilde{P}_{L, N}(y, t),
$$

where $Q_{L, N}$ is an invertible power series in $(y, t)$. By the properties of $\mathcal{U}$ the image of the local map $\left(H_{L}, N\right):\left(\mathbb{C}^{n}, 0\right) \rightarrow\left(\mathbb{C}^{n+1}, 0\right)$ is equal to the germ of the zero set of $\widetilde{P}_{L, N}$. Since $P_{L, N}$ is irreducible, so is $\widetilde{P}_{L, N}$. On the other hand, with any $y$ in a sufficiently small neighbourhood of the origin in $\mathbb{C}^{n}$ we may associate two sets: all roots $\left\{\left(y, t_{1}\right), \ldots,\left(y, t_{r+1}\right)\right\}$ of $\widetilde{P}_{L, N}$ (since $\widetilde{P}_{L, N}$ is a polynomial of degree $r+1$ in $t$ ) and the fiber $H_{L}^{-1}(y)=\left\{z_{1}, \ldots, z_{u}\right\}$ (where $H_{L}$ is treated as a local map $\left(\mathbb{C}^{n}, 0\right) \rightarrow\left(\mathbb{C}^{n}, 0\right)$ ). If additionally $y$ is generic then:

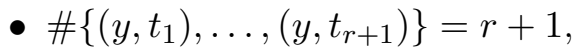

- $u=i_{0}\left(H_{L}\right)$,

- $\left(H_{L}, N\right)$ restricted to $\left\{z_{1}, \ldots, z_{u}\right\}$ is a bijection onto $\left\{\left(y, t_{1}\right), \ldots\right.$, $\left.\left(y, t_{r+1}\right)\right\}$.

As a result we get (7).

By Proposition 3 we have ord $\left(L \circ f-H_{L}\right)>\mathcal{L}_{0}(L \circ f)$. Thus $i_{0}\left(H_{L}\right)=$ $i_{0}(L \circ f)$ by Proposition 2, By (7) and Theorem 11, this ends the proof.

Corollary 6. Let $Q \in \mathbb{C}\{L, N, y, t\}$ be a series of the form

$$
Q(L, N, y, t)=\sum_{j=0}^{\infty} Q_{j}(L, N, y) t^{j} .
$$

If $Q$ is irreducible in $\mathcal{O}_{m n+2 n+1}$ and $Q \circ \Phi=0$ at the level of germs, then

$$
i_{0}(f)=\min \left\{j \in \mathbb{Z}: \operatorname{ord}_{y} Q_{j}=0\right\} .
$$

Proof. Since $P$ and $Q$ are irreducible in $\mathcal{O}_{m n+2 n+1}$ and the germs of the sets $P^{-1}(0)$ and $Q^{-1}(0)$ are equal, $P$ and $Q$ differ by an invertible factor in $\mathcal{O}_{m n+2 n+1}$. Hence Theorem 4 yields the assertion.

2. Semicontinuity of the Łojasiewicz exponent. Let $f:\left(\mathbb{C}^{n}, 0\right) \rightarrow$ $\left(\mathbb{C}^{m}, 0\right)$ be a holomorphic mapping. We say that $F=F_{s}(z)=F(z, s)$ : $\left(\mathbb{C}^{n} \times \mathbb{C}^{k}, 0\right) \rightarrow\left(\mathbb{C}^{m}, 0\right)$ is a deformation of $f$ if $F$ is holomorphic, $F_{0}=f$ and $F_{s}(0)=0$ for $s$ in some neighbourhood of the origin in $\mathbb{C}^{k}$.

In what follows we will use the notion of a complex stratification (or briefly stratification) after [4].

The main result of this section is

THEOREM 7. Let $f:\left(\mathbb{C}^{n}, 0\right) \rightarrow\left(\mathbb{C}^{m}, 0\right)$ be a germ of a holomorphic mapping with an isolated zero and let $F:\left(\mathbb{C}^{n} \times \mathbb{C}^{k}, 0\right) \rightarrow\left(\mathbb{C}^{m}, 0\right)$ be its deformation. Let $U=\bigcup \Gamma_{\nu}^{i}$ be a finite stratification of some sufficiently small neighbourhood $U \subset \mathbb{C}^{k}$ of the origin such that for each stratum $\Gamma_{\nu}^{i}$ the function $\Gamma_{\nu}^{i} \ni s \mapsto i_{0}\left(F_{s}\right) \in \mathbb{Z}$ is constant. Then the function $\Gamma_{\nu}^{i} \ni s \mapsto \mathcal{L}_{0}\left(F_{s}\right) \in \mathbb{Q}$ is 
lower semicontinuous and there exists a finite stratification $\left\{\Sigma_{\mu}^{j}\right\}$, which is a refinement of $\left\{\Gamma_{\nu}^{i}\right\}$, such that the function $\Sigma_{\mu}^{j} \ni s \mapsto \mathcal{L}_{0}\left(F_{s}\right) \in \mathbb{Q}$ is constant for any stratum $\Sigma_{\mu}^{j}$.

In the proof we will need the following

Lemma 8 ([9, Lemma 1]). If $P, Q, R \in \mathbb{C}\{y, t\}$ are series such that

$$
P(y, t)=\sum_{j=0}^{\infty} P_{j}(y) t^{j}, \quad Q(y, t)=\sum_{j=0}^{\infty} Q_{j}(y) t^{j},
$$

ord $R(y, t)=0$ and $Q=P R$, and for some $r \geq 0$ we have ord $P_{j}$, ord $Q_{j}>0$, $j=0, \ldots, r$, then

$$
\min _{j=0}^{r} \frac{\operatorname{ord} P_{j}}{r+1-j}=\min _{j=0}^{r} \frac{\operatorname{ord} Q_{j}}{r+1-j} .
$$

Proof of Theorem 7 . Since the multiplicity and the Eojasiewicz exponent of a local map do not change after perturbation in monomials of orders greater than the multiplicity of the map, we may assume that $F_{s}$ is a polynomial map for any $s$. Let $d=\max \left\{\operatorname{deg} F_{s}: s \in U\right\}$.

Define a mapping $H_{L, s}: \mathbb{C}^{n} \rightarrow \mathbb{C}^{n}$ by

$$
H_{L, s}(z)=L\left(F_{s}(z)\right)+\left(z_{1}^{d^{n}+1}, \ldots, z_{n}^{d^{n}+1}\right),
$$

where $L \in \mathbb{L}(m, n), s \in U$. Set

and let

$$
\mathbb{W}=\mathbb{M}(m, n) \times U
$$

be given by

$$
\Phi: \mathbb{W} \rightarrow \mathbb{W} \times \mathbb{C}
$$

Define $\Phi_{s}: \mathbb{M}(m, n) \rightarrow \mathbb{M}(m, n) \times \mathbb{C}$ for $s \in U$ by

$$
\Phi_{s}(L, N, z)=\left(L, N, H_{L, s}(z), N(z)\right) .
$$

Decreasing $U$ if necessary, we achieve that the mapping $\Phi$ is proper, and consequently, by Remmert's Proper Mapping Theorem, $\Phi(\mathbb{W})$ is an analytic set of pure dimension $m n+2 n+k=\operatorname{dim} \mathbb{W}$. So, for some neighbourhoods $W \subset \mathbb{W}$ and $D \subset \mathbb{W} \times \mathbb{C}$ of the origins and a holomorphic function $Q: D \rightarrow \mathbb{C}$ with an irreducible germ at zero we have $\Phi(W)=\{(w, t) \in D: Q(w, t)=0\}$.

Suppose that the function $Q$ is of the form

$$
Q(L, N, y, s, t)=\sum_{j=0}^{\infty} Q_{j}(L, N, y, s) t^{j},
$$

and denote $Q_{s}(L, N, y, t)=Q(L, N, y, s, t), Q_{j, s}(L, N, y)=Q_{j}(L, N, y, s)$. It is easy to see that $Q_{s}$ is irreducible for $s$ sufficiently close to the origin of $\mathbb{C}^{k}$. Set $W_{s}=\{u \in \mathbb{M}(m, n):(u, s) \in W\}, D_{s}=\{(u, t) \in \mathbb{M}(m, n) \times \mathbb{C}:$ $(u, s, t) \in D\}$. Then $\Phi_{s}\left(W_{s}\right)=\left\{(u, t) \in D_{s}: Q_{s}(u, t)=0\right\}$. Denote by $r_{\nu}^{i}+1$ the multiplicity of $F_{s}$ on $\Gamma_{\nu}^{i}$. From Corollary 6 we have $\operatorname{ord}_{y} Q_{s, j}>0$, $j=0, \ldots, r_{\nu}^{i}$, and $\operatorname{ord}_{y} Q_{s, r_{\nu}^{i}+1}=0$. 
Set

$$
\Delta\left(Q_{s}\right)=\min _{j=0}^{r_{\nu}^{i}} \frac{\operatorname{ord}_{y} Q_{j, s}}{r_{\nu}^{i}+1-j}, \quad s \in \Gamma_{\nu}^{i} .
$$

Observe that the mapping $\Gamma_{\nu}^{i} \ni s \mapsto \Delta\left(Q_{s}\right) \in \mathbb{Q}$ is upper semicontinuous and determines a stratification of $\Gamma_{\nu}^{i}$. Thus, there exists a finite stratification $\left\{\Sigma_{\mu}^{j}\right\}$, which is a refinement of $\left\{\Gamma_{\nu}^{i}\right\}$, such that the function $\Sigma_{\mu}^{j} \ni$ $s \mapsto \Delta\left(Q_{s}\right) \in \mathbb{Q}$ is constant for any stratum $\Sigma_{\mu}^{j}$. On the other hand, by Lemma 8 and Proposition 5 we have $\mathcal{L}_{0}\left(F_{s}\right)=1 / \Delta\left(Q_{s}\right)$ for $s \in U$. This ends the proof.

REMARK 9. It is well known that the stratification $\left\{\Gamma_{\nu}^{i}\right\}$ from Theorem 7 always exists. For example, from Theorem 4 we see that the polynomial $Q_{s}$ used in the proof of Theorem 7 determines such a stratification.

ExAmple 10. Let $F:\left(\mathbb{C}^{2} \times \mathbb{C}^{2}, 0\right) \rightarrow\left(\mathbb{C}^{2}, 0\right)$ be given by the formula

$$
F_{s_{1}, s_{2}}\left(x_{1}, x_{2}\right):=\left(s_{1} x_{1}+x_{2}^{2}, s_{2} x_{2}+x_{1}^{2}\right) \text {. }
$$

For the stratification

$$
\begin{aligned}
& \Gamma_{1}^{2}:=\left\{\left(s_{1}, s_{2}\right): s_{1} s_{2} \neq 0\right\} \\
& \Gamma_{1}^{1} \cup \Gamma_{2}^{1}:=\left\{\left(s_{1}, s_{2}\right): s_{2}=0\right\} \cup\left\{\left(s_{1}, s_{2}\right): s_{1}=0\right\}
\end{aligned}
$$

we have

$$
i_{0}\left(F_{s_{1}, s_{2}}\right)= \begin{cases}1, & \left(s_{1}, s_{2}\right) \in \Gamma_{1}^{2}, \\ 4, & \left(s_{1}, s_{2}\right) \in \Gamma_{1}^{1} \cup \Gamma_{2}^{1} .\end{cases}
$$

If we set $\Sigma_{1}^{2}:=\Gamma_{1}^{2}, \Sigma_{1}^{1}:=\Gamma_{1}^{1} \backslash\{(0,0)\}, \Sigma_{2}^{1}:=\Gamma_{2}^{1} \backslash\{(0,0)\}, \Sigma_{1}^{0}:=\{(0,0)\}$, then

$$
\mathcal{L}_{0}\left(F_{s_{1}, s_{2}}\right)= \begin{cases}1, & \left(s_{1}, s_{2}\right) \in \Sigma_{1}^{2}, \\ 4, & \left(s_{1}, s_{2}\right) \in \Sigma_{1}^{1} \cup \Sigma_{2}^{1}, \\ 2, & \left(s_{1}, s_{2}\right) \in \Sigma_{1}^{0} .\end{cases}
$$

Observe that in this case $F_{s}$ is already a proper polynomial mapping. Using CAS the polynomial $Q_{s}$ is given by

$$
\begin{aligned}
& Q_{s}(N, y, t)=t^{4}+\left(-3 s_{1} s_{2} a_{1} a_{2}-2 y_{2} a_{1}^{2}-2 y_{1} a_{2}^{2}\right) t^{2} \\
& \quad+\left(s_{1} s_{2}^{2} a_{1}^{3}+s_{1}^{2} s_{2} a_{2}^{3}+4 s_{2} y_{1} a_{1}^{2} a_{2}+4 s_{1} y_{2} a_{1} a_{2}^{2}\right) t \\
& \quad-s_{2}^{2} y_{1} a_{1}^{4}-s_{1} s_{2} y_{2} a_{1}^{3} a_{2}-s_{1} s_{2} y_{1} a_{1} a_{2}^{3}-s_{1}^{2} y_{2} a_{2}^{4}+y_{2}^{2} a_{1}^{4}-2 y_{1} y_{2} a_{1}^{2} a_{2}^{2}+y_{1}^{2} a_{2}^{4},
\end{aligned}
$$

where $N(x)=a_{1} x_{1}+a_{2} x_{2}, y=\left(y_{1}, y_{2}\right), s=\left(s_{1}, s_{2}\right)$.

The deformation $F_{s}$ is called multiplicity-constant if the map $s \mapsto i_{0}\left(F_{s}\right)$ has constant finite value.

Corollary 11. If $f$ has an isolated zero and $F:\left(\mathbb{C}^{n} \times \mathbb{C}^{k}, 0\right) \rightarrow\left(\mathbb{C}^{m}, 0\right)$ is a multiplicity-constant deformation of $f$, then there exists $\varepsilon>0$ such that

$$
\mathcal{L}_{0}(f) \leq \mathcal{L}_{0}\left(F_{s}\right) \quad \text { for }|s| \leq \varepsilon .
$$


Acknowledgments. This research was partially supported by the Polish National Science Centre, grant 2012/07/B/ST1/03293.

\section{References}

[1] R. Achilles, P. Tworzewski, and T. Winiarski, On improper isolated intersection in complex analytic geometry, Ann. Polon. Math. 51 (1990), 21-36.

[2] R. N. Draper, Intersection theory in analytic geometry, Math. Ann. 180 (1969), $175-204$.

[3] M. Lejeune-Jalabert and B. Teissier, Séminaire Lejeune-Teissier: Clôture intégrale des idéaux et équisingularité : Chapitre 1, Université Scientifique et Médicale de Grenoble, Laboratoire de mathématiques pures associé au C.N.R.S., 1974.

[4] S. Łojasiewicz, Introduction to Complex Analytic Geometry, Birkhäuser, 1991.

[5] H. Matsumura, Commutative Ring Theory, Cambridge Stud. Adv. Math. 8, Cambridge Univ. Press, Cambridge, 1989.

[6] A. Płoski, Multiplicity and the Łojasiewicz exponent, in: Singularities (Warszawa, 1985), Banach Center Publ. 20, PWN, Warszawa, 1988, 353-364.

[7] A. Płoski, Semicontinuity of the Eojasiewicz exponent, Univ. Iagel. Acta Math. 48 (2010), 103-110.

[8] T. Rodak, Reduction of a family of ideals, Kodai Math. J. 38 (2015), 201-208.

[9] T. Rodak and S. Spodzieja, Effective formulas for the local Eojasiewicz exponent, Math. Z. 268 (2011), 37-44.

[10] A. Różycki, Effective calculations of the multiplicity of polynomial mappings, Bull. Sci. Math. 138 (2014), 343-355.

[11] S. Spodzieja, Multiplicity and the Eojasiewicz exponent, Ann. Polon. Math. 73 (2000), 257-267.

[12] J. Stückrad and W. Vogel, An algebraic approach to the intersection theory, in: The Curves Seminar at Queens, Vol. II (Kingston, Ont., 1981/1982), Queen's Papers in Pure Appl. Math. 61, exp. no. A, Queen's Univ., Kingston, ON, 1982, 32 pp.

[13] B. Teissier, Variétés polaires. I. Invariants polaires des singularités d'hypersurfaces, Invent. Math. 40 (1977), 267-292.

[14] B. Teissier, Polyèdre de Newton jacobien et équisingularité, in: Seminar on Singularities (Paris, 1976/1977), Publ. Math. Univ. Paris VII 7, Univ. Paris VII, Paris, 1980, 193-221.

[15] P. Tworzewski, Intersection theory in complex analytic geometry, Ann. Polon. Math. 62 (1995), 177-191.

Tomasz Rodak, Adam Różycki, Stanisław Spodzieja

Faculty of Mathematics and Computer Science

University of Łódź

S. Banacha 22

90-238 Łódź, Poland

E-mail: rodakt@math.uni.lodz.pl rozycki@math.uni.lodz.pl spodziej@math.uni.lodz.pl 